\title{
First Results from the X-Ray Microscopy Beamline U41-PGM1-XM at BESSY II.
}

$\underline{\text { Peter Guttmann }}^{1, *}$, Stephan Werner ${ }^{1}$, Stefan Rehbein $^{1}$, Catharina Häbel ${ }^{1}$ and Gerd Schneider ${ }^{1}$

1. Helmholtz-Zentrum Berlin für Materialien und Energie, BESSY II, Research Group X-Ray Microscopy, Berlin, Germany.

* Corresponding author, peter.guttmann@helmholtz-berlin.de

The newly designed beamline U41-PGM1-XM at BESSY II for the Helmholtz-Zentrum Berlin (HZB) transmission soft X-ray microscope (TXM) was successfully set up and went in operation in 2017 [1]. During the commissioning of the beamline we determined the spectral resolution, horizontal focus value at the exit slit and the flux for different undulator harmonics. The experimental results meet the values from raytracing calculations. For the horizontal focus at the exit slit position we calculated a FWHM value of $108 \mu \mathrm{m}$ at $510 \mathrm{eV}$ which is in good agreement with the experimental value of $107 \mu \mathrm{m}$. The flux for photon energies higher than $550 \mathrm{eV}$ is now much higher compared to the previous U41-SGM-XM beamline [2] (Fig. 1).

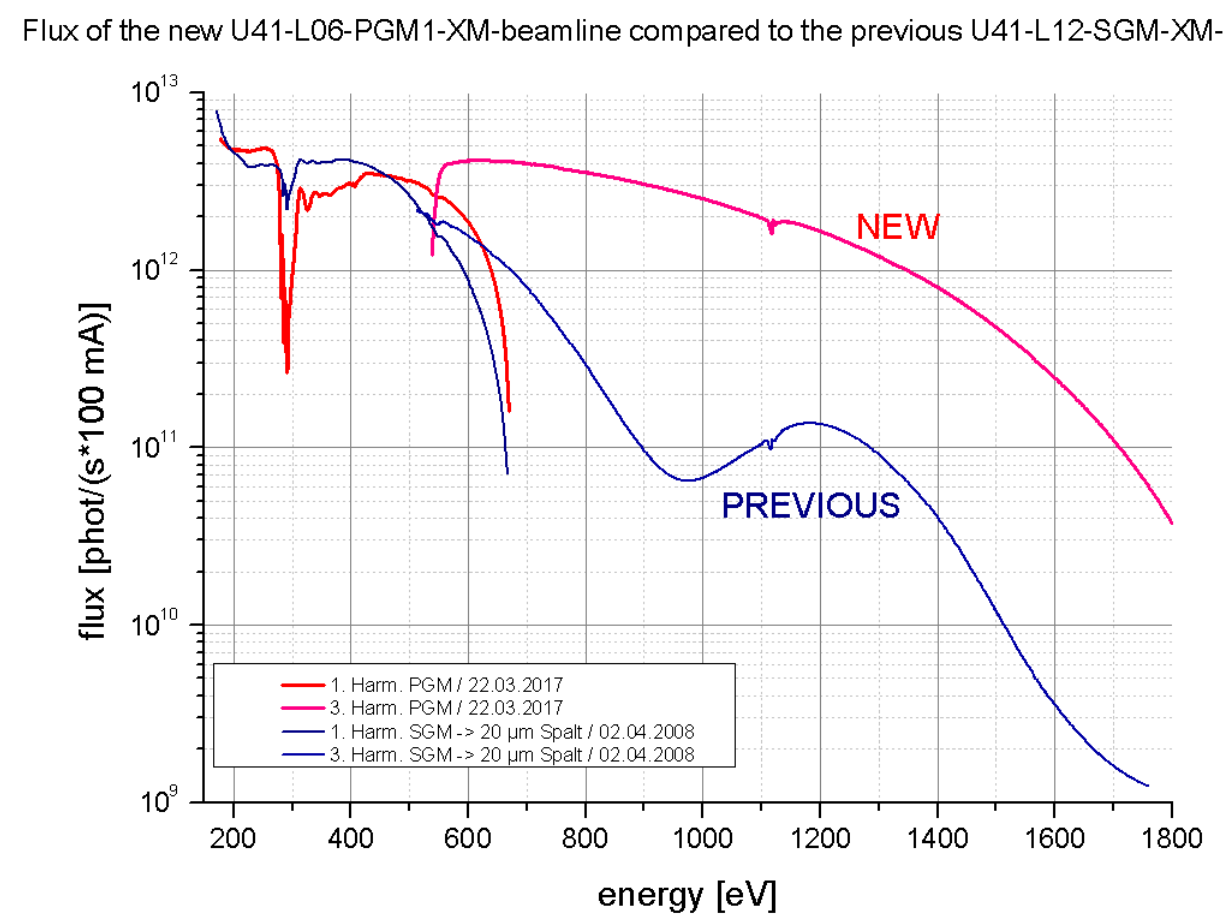

Figure. 1. The measured flux downstream of the exit slit with $20 \mu \mathrm{m}$ opening is shown in comparison to the previous U41-FSGM beamline at location L12. Note the gain especially for the photon energy range larger than $550 \mathrm{eV}$. At both beamlines we have used the same GaAsP-photodiode at the same distance to the exit slit and Keithley device for the measurements.

With the $8001 / \mathrm{mm}$ Au-grating with a blaze angle of $0.93^{\circ}$ we reached a spectral resolution of about 7600 resp. 10600 at $400 \mathrm{eV}$ with $\mathrm{c}_{\mathrm{ff}}$ (constant magnification for fixed focus) $=2.25$ resp. $\mathrm{c}_{\mathrm{ff}}=10$ which is sufficient for the standard nano-tomography applications [2] and NEXAFS-TXM studies [3]. 
The high performance of the new beamline is also demonstrated by the high quality of the X-ray microscopic images. A comparison of images taken from the same Siemens star test pattern with a zone plate having $25 \mathrm{~nm}$ outermost zone width shows clearly artifact free imaging at the new beamline (Fig. 2). Note the improved homogeneity of the illumination. Additionally, a multilayer test pattern lamella demonstrates the high spatial resolution of about $10 \mathrm{~nm}$ (Fig. 3). Third order imaging at higher photon energies, e.g. at $1400 \mathrm{eV}$, can be performed with shorter exposure times compared to the previous beamline (Fig. 3).

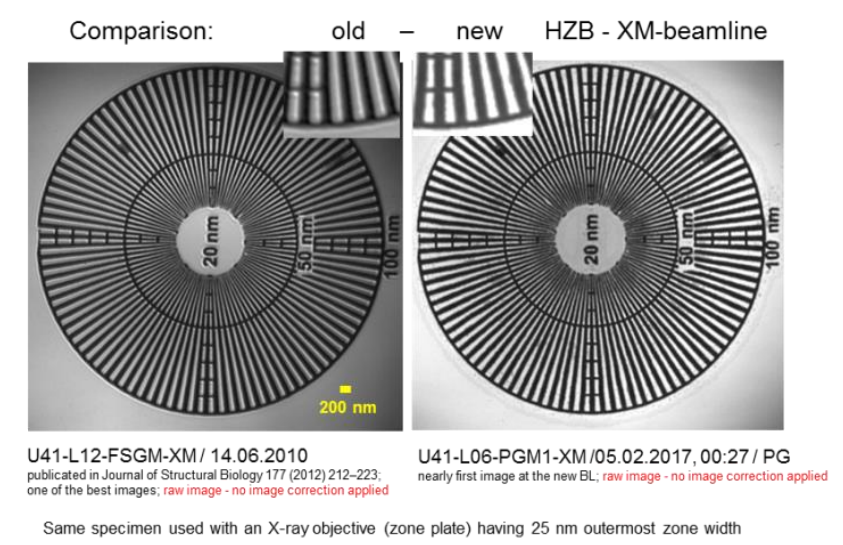

Figure. 2. The beamline optics were designed to illuminate the capillary symmetrically, therefore no imaging artifacts by asymmetrical illumination as we have it before are occurring.
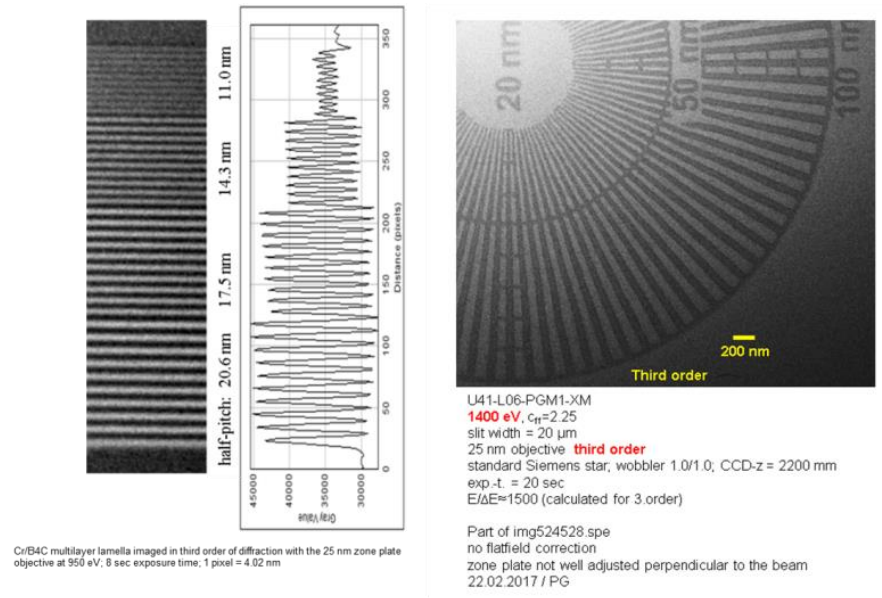

Figure. 3. With test specimens we could demonstrate again a high spatial resolution of about $10 \mathrm{~nm}$ and that we can do imaging at higher photon energies with shorter exposure times.

During first friendly user experiments imaging at $1800 \mathrm{eV}$ could be demonstrated. This is very helpful for studies of e.g. thicker (up to $2 \mu \mathrm{m}$ ) lamellas of materials sciences projects like semiconductor devices. Several NEXAFS-TXM investigations could be carried out underlining the high quality of the beamline [4].

The X-ray microscope using up-to-date EPICS- and QT-based control software is in normal user operation since the second beamtime period of 2017. Now, a special cryo-holder for round standard EM grids can be used for nano-tomography together with a new zone plate objective with $25 \mathrm{~nm}$ outermost zone width for tilt-ranges up to $\pm 60^{\circ}$.

\section{References:}

[1] P. Guttmann et al, The New HZB X-Ray Microscopy Beamline U41-PGM1-XM at BESSY II, this conference

[2] G. Schneider et al, J. Struct. Biol. 177 (2012), 212-223

[3] P. Guttmann et al, Nature Photonics 6 (2012), 25-29

[4] C. Bittencourt et al, this conference 